\title{
解构重组：从头构建羟基取代苯并呋喃的新策略
}

\author{
张梓曜焦 宁*
}

(北京大学天然药物及仿生药物国家重点实验室 北京 100191)

\section{Deconstructive Reorganization: De Novo Synthesis of Hydroxylated Benzofurans}

\author{
Zhang, Ziyao Jiao, Ning* \\ (State Key Laboratory of Natural and Biomimetic Drugs, Peking University, Beijing 100191)
}

羟基取代苯并呋喃是一种广泛存在于天然产物和 生物活性分子中的结构. 目前, 官能团化苯并呋喃的合 成通常使用两种策略: (1)过渡金属催化的 $\mathrm{sp}^{2}$ 碳氢键官 能团化; (2)通过环化反应构建苯环或呋喃环. 这两种策 略都存在一定的局限性. 碳氢键官能团化是一种强大的 策略, 近年来发展出多种导向基协助的吲哚 $4 \sim 7$ 位的 碳氢键官能团化反应 ${ }^{[1]}$. 但是由于呋喃环的氧原子无法 引入导向基团, 因此无法活化苯并呋喃的 6,7 位的碳氢 键. 环化反应构建苯并呋喃骨架策略众多, 但是要在其 苯环的 4 7 位直接引入不同取代模式的羟基仍然是一 个巨大的挑战 ${ }^{[2]}$. 通常引入羟基时需要保护和脱保护, 或者采用还原的策略将酮或醌还原为羟基, 这就造成了 步骤上的浪费以及经济性的降低.

过渡金属催化的羰基亲核进攻炔烃构建官能团化 的氧杂环近年来受到研究人员的广泛关注. 尤其是炔羰 基化合物, 比如炔酮和炔醛, 是合成呋喃骨架的有效前 体. 根据 Baldwin 规则, 炔羰基化合物的分子内 5-endodig 亲核进攻可以高效地构建呋喃环. 2004 年, Larock 课 题组 ${ }^{[3]}$ 采用这种策略, 利用三氯化金高效催化炔酮的分 子内关环反应, 合成了高度取代的呋喃衍生物. 而后刘 元红 ${ }^{[4]}$ 、张俊良 ${ }^{[5]}$ 和 Schmalz 等 ${ }^{[6]}$ 分别发展了一系列以 金、钉等 $\pi$ 酸活化炔烃, 进一步串联构建并环结构的策

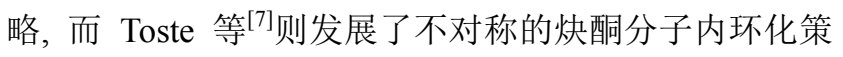
略.

华南理工大学化学化工学院祝诗发课题组在 $\pi$ 酸活 化炔烃及后续转化方面开展了一系列工作. 在此基础 上, 他们提出了一种新颖的解构重组策略(Deconstructive Reorganization), 利用廉价的工业产品曲酸(kojic acid)和麦芽酚 (maltol)的炔基化衍生物为原料, 经过炔 酮分子内 5-endo-dig 关环生成吡喃鎓并呋喃环中间体, 外加亲核试剂进攻氧鎓离子打开吡喃环，再通过串联 aldol 缩合脱水关环构建苯环, 最终实现了羟基苯并呋 喃骨架的直接构建 $(\text { 图 } 1)^{[8]}$. 该反应使用廉价工业品的衍 生物作为原料, 无需氧化还原过程, 底物来源广泛, 条 件温和, 相比先前的羟基苯并呋喃合成具有较大的优 势.

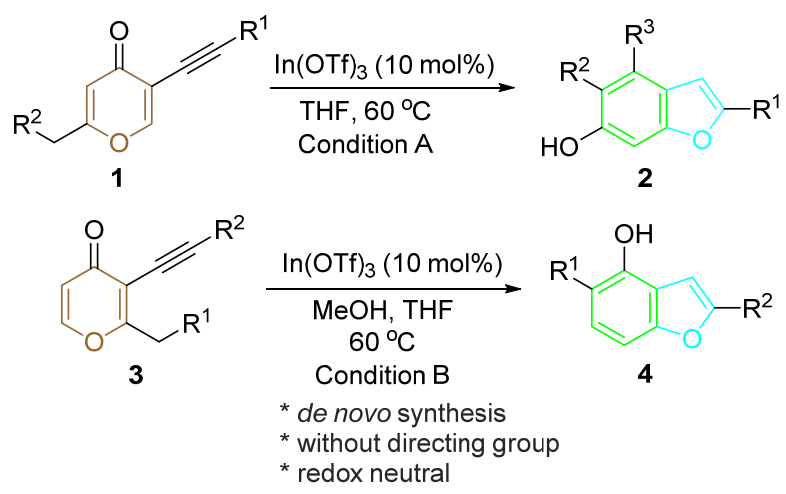

图 1 差基取代的苯并呋喃的从头合成

Figure 1 de novo synthesis of hydroxylated benzofuran

条件篮选显示, 使用 $\operatorname{In}(\mathrm{OTf})_{3}$ 作为 $\pi$ 酸, 在四氢呋 喃中 $60{ }^{\circ} \mathrm{C}$ 反应 $12 \mathrm{~h}$, 可以以较高的收率得到目标产物. 以曲酸衍生物作为底物, 水作亲核试剂, 可以专一地得 到 5,6 位羟基化的苯并呋喃; 以麦芽酚衍生物作为底物, 甲醇作为亲核试剂, 可以专一地得到 4 位羟基化的苯并 呋喃.

使用不同取代基的底物, 可以顺利地实现多样性官 能团化的苯并呋喃的合成. 值得一提的是, 使用他们的

* Corresponding author. E-mail: jiaoning@bjmu.edu.cn. Published online April 9, 2020. 


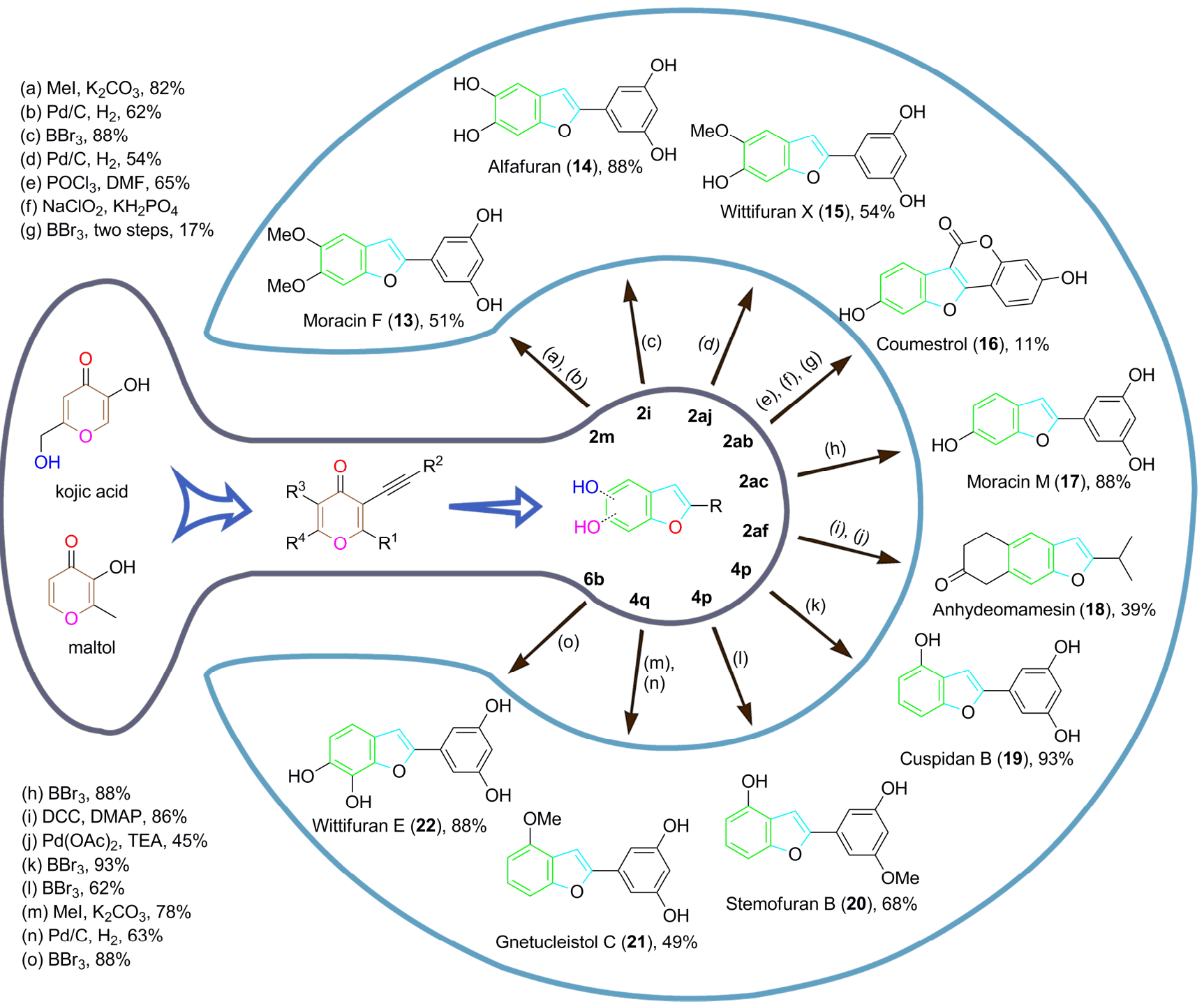

图 2 应用于合成多种含羟基化苯并呋喃结构的天然产物

Figure 2 Application in collective total synthesis of hydroxylated benzofuran-containing natural products

方法, 再进行 $1 \sim 3$ 步简单转化后, 可以合成一系列具有 生物活性的天然产物(图 2). 另外他们运用结构重组的 策略进行了天然产物 8-epi-Liphgal 的全合成. 相比传统 的仿生合成策略, 他们的方法路线更短并具有更好的立 体选择性 ${ }^{[9]}$.

机理研究表明, 在羰基进攻炔烃关环后, 曲酸衍生 的吡喃环受水亲核进攻, 开环生成 $\alpha$-羊基酮和醛直接 aldol 缩合; 麦芽酚衍生物的吡喃环在甲醇的亲核进攻 下开环形成缩醛和酮, 而后加入盐酸脱除甲醇进一步 aldol 缩合.

总之, 祝诗发研究团队发展了一种全新的解构重组 策略, 利用大宗工业原料高效地从头合成羟基化苯并呋 喃, 在天然产物全合成中做了大量的应用. 他们的工作 丰富了构建羟基化杂环的方法学, 有望在天然产物及药 物合成领域发挥重要的作用.

\section{References}

[1] Luo, J.; Xu, X.; Zheng, J. Chin. J. Org. Chem. 2018, 38, 363 (in Chinese). (骆钧飞, 徐星, 郑俊良, 有机化学, 2018, 38, 363.)

[2] Zhang, W.; Xu, W.; Zhang, F.; Li, Y. Chin. J. Org. Chem. 2019, 39, 1277 (in Chinese). (张文生，许文静，张斐，李炎，有机化学, 2019, 39, 1277.)

[3] Yao, T.; Zhang, X.; Larcok, R. C. J. Am. Chem. Soc. 2004, 126, 11164.

[4] Xie, X.; Du, X.; Chen, Y.; Liu, Y. J. Org. Chem. 2011, 76, 9175.

[5] Zhao, W.; Zhang, J. Chem. Commun. 2010, 46, 4384.

[6] Zhang, J.; Schmalz, H. G. Angew. Chem., Int. Ed. 2006, 45, 6704.

[7] Rauniyar, V.; Wang, Z. J.; Burks, H. E.; Toste, F. D. J. Am. Chem. Soc. 2011, 133, 8486.

[8] Zhang, L.; Cao, T.; Jiang, H.; Zhu, S. Angew. Chem., Int. Ed. 2020, $59,4670$.

Marion, F.; Williamms, D. E.; Patrick, B. O.; Hollander, I.; Mallon, R.; Kim, S. C.; Roll, D. M.; Feldberg, L.; Van Soest, R.; Anderson, R. J. Org. Lett. 2006, 8. 321.

(Cheng, F.) 